MS-TP-08-01

\title{
Interfacial roughening in field theory
}

\author{
Michael H. Köpf and Gernot Münster*
}

January 23, 2008

\begin{abstract}
In the rough phase, the width of interfaces separating different phases of statistical systems increases logarithmically with the system size. This phenomenon is commonly described in terms of the capillary wave model, which deals with fluctuating, infinitely thin membranes, requiring ad hoc cut-offs in momentum space. We investigate the interface roughening from first principles in the framework of the Landau-Ginzburg model, that is renormalized field theory, in the one-loop approximation. The interface profile and width are calculated analytically, resulting in finite expressions with definite coefficients. They are valid in the scaling region and depend on the known renormalized coupling constant.
\end{abstract}

KEY WORDS: Interfaces, field theory

\section{Introduction}

Interface roughening is a phenomenon which has attracted interest of experimental and theoretical investigators, see e.g. 1, 2, 3, 4, 5, 6, 7, since its discovery [8]. It is displayed by interfaces, separating different coexisting phases or substances of a system of statistical physics, in a range of temperatures $T_{R}<T<T_{c}$ between the roughening temperature $T_{R}$ and the critical temperature $T_{c}$. Roughening manifests itself in a characteristic dependence of the interface width on the system size. For an interface of diameter $L$ the width increases logarithmically with $L$ in the rough phase, whereas it remains constant of the order of the correlation length $\xi$ for temperatures below $T_{R}$.

This effect is commonly described theoretically in terms of the capillary wave model or drumhead model 8 . In this model the interface is represented in an idealized way by an infinitely thin fluctuating membrane, so

\footnotetext{
*Institut für Theoretische Physik, Universität Münster, Wilhelm-Klemm-Str. 9, D-48149 Münster, Germany; e-mail: munsteg@uni-muenster.de
} 
that the instantaneous microscopic interface profile is a sharp step function between the two phases. Nevertheless, in the thermal average the capillary wave fluctuations produce a continuous density profile with a finite width $w$, which can be shown to be given by an integral over all wave-numbers of the fluctuations, which is essentially of the form

$$
w^{2}=\frac{1}{2 \pi \sigma} \int_{k_{\min }}^{k_{\max }} d k k^{D-4},
$$

where $D$ is the number of dimensions of space and $\sigma$ is the interface tension. A natural lower limit on the wave numbers is given by the system size,

$$
k_{\min }=\frac{\text { const. }}{L} \text {. }
$$

In order to avoid the divergence of the integral, an upper cut-off $k_{\max }$ has to be introduced. As there should be no waves with wavelength smaller than the intrinsic width of the physical interface, the upper cut-off is taken to be of the order of the inverse correlation length. In the case of $D=3$, considered here, one obtains

$$
w^{2}=\frac{1}{2 \pi \sigma} \ln \frac{L}{c \xi}
$$

with an unknown constant $c$. The logarithmic increase with $L$ is due to the contribution of capillary waves with long wavelengths near the system size $L$.

Complementary to the capillary wave model is the mean field description of interfaces. In mean field theory and its field theoretic refinements, interfaces possess an intrinsic continuous profile with a well-defined width, which is proportional to the bulk correlation length and does not depend on the system size.

Mean field and capillary wave theory can be combined in the "convolution approximation" 9, 10. In this picture the intrinsic profile describes the interface on a microscopic scale of the order of the correlation length, while capillary wave theory describes the macroscopic interface fluctuations of wavelengths much larger than the correlation length. The intrinsic profile is thus centered around a two-dimensional surface subject to capillary wave fluctuations. In the convolution approximation the square of the resulting total interface width is obtained as a sum of the intrinsic part and the capillary wave contribution,

$$
w^{2}=c_{1} \xi^{2}+\frac{1}{2 \pi \sigma} \ln \frac{L}{c_{2} \xi} .
$$

The description of rough interfaces by means of the capillary wave model and the convolution approximation is unsatisfactory for different reasons. 
First of all, it has so far not been possible to define the concept of an intrinsic interface profile and width unambiguously outside a given theory. In experiments or Monte Carlo simulations of systems with interfaces, the observed interface profile and width are the total ones, including the effects of the intrinsic structure as well as of the capillary waves, and there is no clear way to separate the intrinsic structure from the effects of capillary waves. Secondly, the models sketched above contain ad hoc constants, whose numerical values are arbitrary and cannot be fixed unambiguously within the models.

In this article we investigate the profile and width of rough interfaces in a coherent approach from first principles. Statistical systems with coexisting phases, separated by interfaces, are described in the framework of the field theoretic version of the Landau-Ginzburg model, including fluctuations on all length scales. No cut-off on wave-numbers is introduced. For explicit calculations we employ the one-loop approximation. It should be noted, however, that an extension to arbitrary higher loop orders is possible in principle. The interface profile, resulting from the calculation, shows the expected logarithmic broadening with the system size $L$. We obtain analytical results for the numerical coefficients, which are fixed unambiguously in this approach.

Interfaces have been studied before in the framework of field theory by other authors. In [11, 12 the profile is calculated to first order in the $\epsilon$ expansion, where $D=4-\epsilon$ and an extrapolation to $\epsilon=1$ is necessary. The $\epsilon$-expansion is an expansion around the four-dimensional case. As can be seen from Eq. (1), in four dimensions the contribution of long-wavelength modes converges and no roughening is present. This has the consequence that within the $\epsilon$-expansion, even after extrapolation to $D=3$ dimensions, roughening effects do not show up, as is well known. The calculation of [11] is extended to include the effects of an external field in [13.

Our calculations are performed in $D=3$ physical dimensions in contrast to the $\epsilon$-expansion. The three-dimensional approach is based on a systematic expansion in a dimensionless coupling [14, 15]. Ultraviolet divergences are treated by dimensional regularization $(D=3-\epsilon)$, which does not vitiate the fact that the results for physical quantities strictly refer to $D=3$ dimensions. This is also seen explicitly by the fact that the calculation reveals the typical roughening effects. Renormalization of the three-dimensional field theory is performed in the scheme used in [16] to two-loop order, employing the results of [17, 18.

A three-dimensional study has previously been done in 19 , where the interface profile is considered in $D=3$ dimensions at one-loop order in the presence of an external gravitational field. A functional form of the profile is given, including capillary wave effects. The dependence on the system size is, however, not considered. We shall compare our results with the 
ones of 19 below.

\section{Interfaces in field theory}

In the framework of field theory, the system under consideration, possessing interfaces, is described by an order parameter field $\phi(x)$ representing the difference between the concentrations of the two coexisting phases. The physics of the system is governed by the Landau-Ginzburg Hamiltonian [20]

$$
H[\phi]=\int d^{3} x \mathcal{H}(\phi(x))
$$

with the Hamiltonian density

$$
\mathcal{H}(\phi)=\frac{1}{2} \partial_{\mu} \phi \partial_{\mu} \phi+V_{0}(\phi)
$$

In the situation with interfaces the potential is of the double-well type,

$$
V_{0}(\phi)=\frac{g_{0}}{4 !}\left(\phi^{2}-v_{0}^{2}\right)^{2} .
$$

Mean field theory amounts to the classical approximation where fluctuations are neglected. The minima of the potential then correspond to the two homogeneous phases. The mean field correlation length $\xi_{0}$ is defined through the second moment of the correlation function in the mean field approximation. It is given by the second derivative of the potential in its minima:

$$
\xi_{0}^{2}=\left(V_{0}^{\prime \prime}\left(v_{0}\right)\right)^{-1}=\frac{3}{g_{0} v_{0}^{2}} .
$$

With the bare mass $m_{0}$, defined by

$$
m_{0}=\frac{1}{\xi_{0}}
$$

the Hamiltonian density can be written as

$$
\mathcal{H}(\phi)=\frac{1}{2} \partial_{\mu} \phi \partial_{\mu} \phi-\frac{m_{0}^{2}}{4} \phi^{2}+\frac{g_{0}}{4 !} \phi^{4}+\frac{3}{8} \frac{m_{0}^{4}}{g_{0}} .
$$

The simplest description of interfaces is also based on mean field theory [21]. In this approximation the interface profile is given by minimization of the Hamiltonian $H$ with boundary conditions appropriate for an interface. The corresponding field equation

$$
\frac{\delta H}{\delta \phi(x)}=0
$$


leads to the differential equation

$$
\Delta \phi-V_{0}^{\prime}(\phi)=0 .
$$

If we choose the interface to be perpendicular to the $z$-axis, we find the typical hyperbolic tangent profile 22]

$$
\phi_{0}^{\left(z_{0}\right)}(z)=v_{0} \tanh \left(\frac{z-z_{0}}{2 \xi_{0}}\right) .
$$

Its width is proportional to the mean field correlation length $\xi_{0}$. The parameter $z_{0}$ specifies the location of the interface.

Essential for a field theoretic treatment, as being considered in this article, are corrections to mean field theory coming from fluctuations of the order parameter field. They can be calculated systematically in renormalized perturbation theory. The fluctuations result in different modifications of the mean field result, as will be considered in detail below. First of all, higher order corrections change the form of the profile from the tanh-function to a different function. Secondly, renormalization of the parameters $v_{0}$ and $\xi_{0}$ becomes necessary and, as a result, the mean field correlation length $\xi_{0}$ is replaced by the physical correlation length $\xi$, which diverges near the critical point with a characteristic exponent $\nu$. Finally, long-wavelength fluctuations lead to the roughening phenomenon, which implies a broadening of the interface, such that its width depends logarithmically on the system size and diverges in the limit of an infinite system.

The partition function for the system with an interface can be written as a functional integral of the form

$$
Z=\int \mathcal{D} \varphi \exp \left(-H\left[\phi_{0}+\varphi\right]\right),
$$

where $\phi_{0}(z)$ is a classical interface solution as given above and $\varphi(x)$ denotes the fluctuations around it. The Hamiltonian density, expressed in terms of $\varphi$, reads

$$
\mathcal{H}\left(\phi_{0}+\varphi\right)=\mathcal{H}\left(\phi_{0}\right)+\frac{1}{2} \varphi(x) K \varphi(x)+\frac{g_{0}}{3 !} \phi_{0}(x) \varphi^{3}(x)+\frac{g_{0}}{4 !} \varphi^{4}(x) .
$$

Here the operator $K$ is given by

$$
K=-\Delta-\frac{m_{0}^{2}}{2}+\frac{g_{0}}{2} \phi_{0}^{2}(x),
$$

where $\Delta$ is the Laplacean.

In the loop expansion the quadratic terms in $\mathcal{H}$ are treated by means of Gaussian functional integrals, and the higher order terms are taken into account by Taylor expansions. 
The spectrum of $K$ is known analytically [23. We have to employ it for our calculation and give details below. At this point we would like to draw the attention to the fact that $K$ has a single zero mode

$$
K \psi(x)=0 .
$$

The zero mode of the fluctuation operator is directly related to translations of the interface, as parameterized by the parameter $z_{0}$. For every value of this parameter, the function $\phi_{0}^{\left(z_{0}\right)}$ is a solution of the classical field equation. This implies that

$$
\psi(z)=\frac{d \phi_{0}^{\left(z_{0}\right)}(z)}{d z_{0}}
$$

is a zero mode of $K$.

The existence of a zero mode requires to treat the corresponding fluctuations, which are proportional to $\psi$, separately from the remaining Gaussian integrals in the functional integrals. This is done by the method of collective coordinates 24]. The collective coordinate in question is $z_{0}$. In the Gaussian integral it is set to an arbitrary value, which we choose to be $z_{0}=0$, and the fluctuations are restricted to the space $\mathcal{N}_{\perp}$ of functions orthogonal to the zero mode $\psi$ :

$$
\int d^{3} x \varphi(x) \psi(x)=0 .
$$

When expectation values in the presence of an interface are calculated, integration over $z_{0}$ would imply averaging over all translations of the interface, leading to translationally invariant results. In case of the interface profile, however, this is obviously not appropriate, since one is interested in the profile function relative to the position of the interface. Therefore integration over $z_{0}$ has to be omitted, leaving us with Gaussian integrals over $\mathcal{N}_{\perp}$. So the interface profile is given by

$$
\phi_{c}(x)=\phi_{0}(x)+\phi_{f}(x)
$$

with

$$
\phi_{f}(x)=\langle\varphi(x)\rangle=\frac{1}{Z^{\prime}} \int_{\mathcal{N}_{\perp}} \mathcal{D} \varphi \varphi(x) \exp \left(-H\left[\phi_{0}+\varphi\right]\right) .
$$

\section{The profile equation}

For functional integrals over the fluctuation field $\varphi \in \mathcal{N}_{\perp}$ Feynman rules can be set up analogously to the usual case. The propagator and vertices can be read off the Hamiltonian (15). The propagator is the inverse of the fluctuation operator restricted to $\mathcal{N}_{\perp}$ :

$$
K^{\prime}=\left.K\right|_{\mathcal{N}_{\perp}} \text {. }
$$


There are three-point and four-point vertices, given by

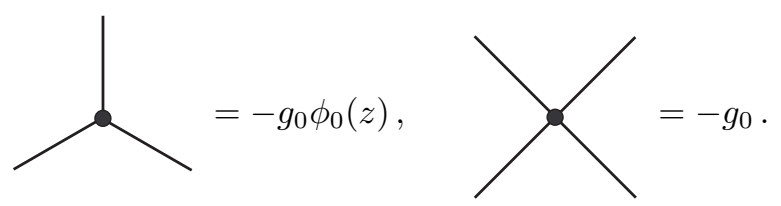

The fluctuation part the interface profile gets contributions from all orders of the loop expansion:

$$
\phi_{f}(x)=\phi_{1}(x)+\phi_{2}(x)+\ldots
$$

In the one-loop approximation, which we employ, the Feynman diagram contributing to the profile function

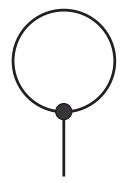

leads to

$$
\phi_{1}(x)=-\frac{g_{0}}{2} \int d^{3} x^{\prime} K^{\prime-1}\left(x, x^{\prime}\right) K^{\prime-1}\left(x^{\prime}, x^{\prime}\right) \phi_{0}\left(x^{\prime}\right) .
$$

Here the kernel of the inverse operator $K^{\prime-1}$ enters. It would be possible to calculate $\phi_{1}$ from this expression. It is, however, more convenient to obtain it as a solution of a differential equation. Acting with the operator $K$ on Eq. (24), we obtain the profile equation

$$
K \phi_{1}(x)+\frac{g_{0}}{2} K^{\prime-1}(x, x) \phi_{0}(x)=0 .
$$

In order to solve this equation we need the explicit form of $K^{\prime-1}(x, x)$, which is discussed below.

An alternative derivation of the profile equation is based on the so-called effective action $\Gamma[\Phi]$, which is a functional of a field $\Phi(x) . \Gamma[\Phi]$ is obtained by Legendre transformation from the free energy in the presence of a nonconstant external field. For constant $\Phi$ the effective action reduces to the Gibbs potential. For a definition and discussion see e.g. 25. Calculating $\Gamma[\Phi]$ in the one-loop approximation and finding the interface profile $\phi(x)$ as a stationary point of $\Gamma$,

$$
\frac{\delta \Gamma}{\delta \Phi(x)}=0,
$$

again leads to Eq. (25). 


\section{Solution of the profile equation}

The inverse of the fluctuation operator $K^{\prime}$ at coinciding arguments, which enters the profile equation, can be obtained by means of the spectral representation. $K$ is the sum of the negative two-dimensional Laplacean and a one-dimensional Schrödinger operator $\tilde{K}$,

$$
K=-\Delta^{(2)}+\tilde{K},
$$

where

$$
\tilde{K}=-\partial_{z}^{2}+m_{0}^{2}-\frac{3 m_{0}^{2}}{2} \operatorname{sech}^{2}\left(\frac{m_{0}}{2} z\right) .
$$

The negative Laplacean on the $L \times L$ square has eigenvalues

$$
k^{2} \quad \text { with } \quad \vec{k}=\frac{2 \pi}{L} \vec{n}, \quad \vec{n} \in \mathbb{Z}^{2},
$$

and corresponding eigenfunctions

$$
\varphi_{\vec{n}}(\vec{x})=L^{-1} \mathrm{e}^{\mathrm{i} \frac{2 \pi}{L} \vec{n} \cdot \vec{x}}, \quad \vec{x} \in[0, L]^{2} .
$$

The spectrum of $\tilde{K}$ is known exactly [23]. It consists of two discrete eigenvalues

$$
\begin{gathered}
\omega^{(0)}=0, \quad \psi_{0}(z)=\sqrt{\frac{3 m_{0}}{8}} \operatorname{sech}^{2}\left(\frac{m_{0}}{2} z\right), \\
\omega^{(1)}=\frac{3}{4} m_{0}^{2}, \quad \psi_{1}(z)=\sqrt{\frac{3 m_{0}}{4}} \tanh \left(\frac{m_{0}}{2} z\right) \operatorname{sech}\left(\frac{m_{0}}{2} z\right),
\end{gathered}
$$

and a continuum

$$
\omega_{p}=m_{0}^{2}+p^{2} \quad \text { with } p \in \mathbb{R}
$$

$$
\psi_{\omega_{p}}(z)=\mathcal{N}_{p} \mathrm{e}^{\mathrm{i} p z}\left[2 p^{2}+\frac{m_{0}^{2}}{2}-\frac{3}{2} m_{0}^{2} \tanh ^{2}\left(\frac{m_{0}}{2} z\right)+3 \mathrm{i} m_{0} p \tanh \left(\frac{m_{0}}{2} z\right)\right]
$$

with the normalization factor

$$
\mathcal{N}_{p}=\left(2 \pi\left(4 p^{4}+5 m_{0}^{2} p^{2}+m_{0}^{4}\right)\right)^{-\frac{1}{2}} .
$$

The spectrum of $K$ is thus given by

$$
\lambda_{\vec{n} \omega}=\frac{4 \pi^{2}}{L^{2}} n^{2}+\omega, \quad \Psi_{\vec{n} \omega}(x)=\varphi_{\vec{n}}(\vec{x}) \psi_{\omega}(z),
$$

where $\omega$ runs through the eigenvalues of $\tilde{K}$. The zero mode, discussed above, is represented by $\Psi_{\overrightarrow{0} 0}$. 
In terms of the spectrum we write

$$
K^{\prime-1}(x, x)=\sum_{\lambda} \psi_{\lambda}(x) \psi_{\lambda}^{*}(x) \frac{1}{\lambda} .
$$

Inserting the explicit expressions, we obtain

$K^{\prime-1}(x, x)=C_{0}+\left(C_{1}-C_{2}+C_{4}\right) \operatorname{sech}^{4}\left(\frac{m_{0}}{2} z\right)+\left(C_{2}+C_{3}\right) \operatorname{sech}^{2}\left(\frac{m_{0}}{2} z\right)$,

where the coefficients $C_{i}$ are

$$
\begin{aligned}
C_{0} & =\frac{1}{2 \pi} \int d p \sum_{\vec{n}} \frac{1}{4 \pi^{2} n^{2}+\left(m_{0}^{2}+p^{2}\right) L^{2}}, \\
C_{1} & =\frac{3 m_{0}}{8} \sum_{\vec{n} \neq \overrightarrow{0}} \frac{1}{4 \pi^{2} n^{2}}, \\
C_{2} & =\frac{3 m_{0}}{4} \sum_{\vec{n}} \frac{1}{4 \pi^{2} n^{2}+\frac{3}{4} m_{0}^{2} L^{2}}, \\
C_{3} & =-3 m_{0}^{2} \int d p \mathcal{N}_{p}^{2} \sum_{\vec{n}} \frac{m_{0}^{2}+p^{2}}{4 \pi^{2} n^{2}+\left(m_{0}^{2}+p^{2}\right) L^{2}}, \\
C_{4} & =\frac{9}{4} m_{0}^{4} \int d p \mathcal{N}_{p}^{2} \sum_{\vec{n}} \frac{1}{4 \pi^{2} n^{2}+\left(m_{0}^{2}+p^{2}\right) L^{2}} .
\end{aligned}
$$

These expressions are divergent and have to be regularized, as discussed below.

With the explicit form of $K^{\prime-1}(x, x)$ at hand, the solution of the profile equation is found as

$$
\begin{aligned}
\phi_{1}(z)= & \frac{g_{0} v_{0}}{2 m_{0}^{2}}\left\{C_{0} \tanh \left(\frac{m_{0}}{2} z\right)\right. \\
& -\left[\frac{2}{3}\left(C_{1}-C_{2}+C_{4}\right) \tanh \left(\frac{m_{0}}{2} z\right)\right. \\
& \left.\left.-\left(C_{0}+C_{2}+C_{3}\right) \frac{m_{0}}{2} z\right] \operatorname{sech}^{2}\left(\frac{m_{0}}{2} z\right)\right\} .
\end{aligned}
$$

Written in this way, the expression for the profile contains the divergent coefficients $C_{i}$ as well as the bare parameters $g_{0}, m_{0}$ and $v_{0}$. In order to arrive at a finite expression in terms of physical parameters, renormalization has to be performed.

The divergences have to be treated in some regularization scheme. We choose to employ dimensional regularization in $D=3-\epsilon$ dimensions. 
It should be noted that this does not amount to an $\epsilon$-expansion, since after renormalization $\epsilon$ is sent to zero, whereas in the $\epsilon$-expansion one has $D=4-\epsilon$ and the results have to be extrapolated to $\epsilon=1$. So our use of dimensional regularization does not vitiate the fact that the results for physical quantities strictly refer to $D=3$ dimensions. Using other regularization schemes, like Pauli-Villars, would lead to the same final results.

We adopt the renormalization scheme used in 16 to one-loop order. The renormalized mass $m_{R}=1 / \xi$ is equal to the inverse correlation length $\xi$, which in turn is defined through the second moment of the correlation function. The field $\phi$ and its expectation value $v$ are renormalized according to

$$
\phi_{R}(x)=\frac{1}{\sqrt{Z_{R}}} \phi(x), \quad v_{R}=\frac{1}{\sqrt{Z_{R}}} v,
$$

where $Z_{R}$ is the usual field renormalization factor. The renormalized coupling is specified as in [26] through

$$
g_{R}=\frac{3 m_{R}^{2}}{v_{R}^{2}} .
$$

In addition we define a dimensionless renormalized coupling according to

$$
u_{R}=\frac{g_{R}}{m_{R}^{4-D}} .
$$

Employing the relations given in [17, 18, the bare quantities $m_{0}$ and $g_{0}$ are expressed in terms of their renormalized counterparts.

The coefficients $C_{i}$ are evaluated in the same scheme. Leaving out the lengthy details, we quote the results

$$
\begin{aligned}
C_{0} & =-\frac{m_{0}}{4 \pi} \\
C_{2}+C_{3} & =\frac{3 m_{0}}{16 \pi} \ln 3 \\
C_{1}-C_{2}+C_{4} & =\frac{3 m_{0}}{16 \pi}\left(-\alpha+\ln \left(m_{0} L\right)\right)
\end{aligned}
$$

with

$$
\alpha=\ln \left(\frac{3 \Gamma^{2}(1 / 4)}{2 \sqrt{\pi}}\right)-\gamma \approx 1.832,
$$

where $\gamma \approx 0.577$ is Euler's constant.

The coefficients $C_{i}$ contain additional terms decaying exponentially fast with $L$, which we neglect here.

Inserting everything into the expression for the interface profile yields the renormalized interface profile $\phi_{R}(z)$ depending on the parameters $m_{R}$ 
and $u_{R}$. In this expression the divergences are cancelled, as they should. Expanding consistently in powers of $u_{R}$ up to the first order, we obtain

$$
\begin{aligned}
\phi_{R}(z)=v_{R}\{ & \tanh \left(\frac{m_{R}}{2} z\right) \\
& +\frac{u_{R}}{16 \pi}\left(\alpha-\ln \left(m_{R} L\right)\right) \tanh \left(\frac{m_{R}}{2} z\right) \operatorname{sech}^{2}\left(\frac{m_{R}}{2} z\right) \\
& \left.-\frac{u_{R}}{32 \pi}\left(3 \ln 3-\frac{13}{4}\right) \frac{m_{R}}{2} z \operatorname{sech}^{2}\left(\frac{m_{R}}{2} z\right)\right\},
\end{aligned}
$$

which is the central result of this article. Asymptotically, for $|z| \rightarrow \infty$, the profile approaches the bulk expectation value $\pm v_{R}$, corresponding to the pure phases of the system, as it should be. Note that the profile depends logarithmically on the system size $L$, revealing the effect of capillary wave fluctuations. It is this term, depending on $m_{R} L$, which represents the deviation from the Fisk-Widom [27] scaling form $\phi\left(m_{R} z\right)$.

In order to illustrate the characteristics of the interface profile, we have to specify a numerical value for the dimensionless renormalized coupling $u_{R}$. In the vicinity of the critical point the coupling varies only slowly and is close to the universal fixed point value $u_{R}^{*}=14.3(1)$, see [28 for a discussion of numerical and field-theoretical estimates. Therefore we take $u_{R}=14.3$ in the plot. The interface profile according to Eq. (52) is displayed in Fig.1 for different values of $m_{R} L$.

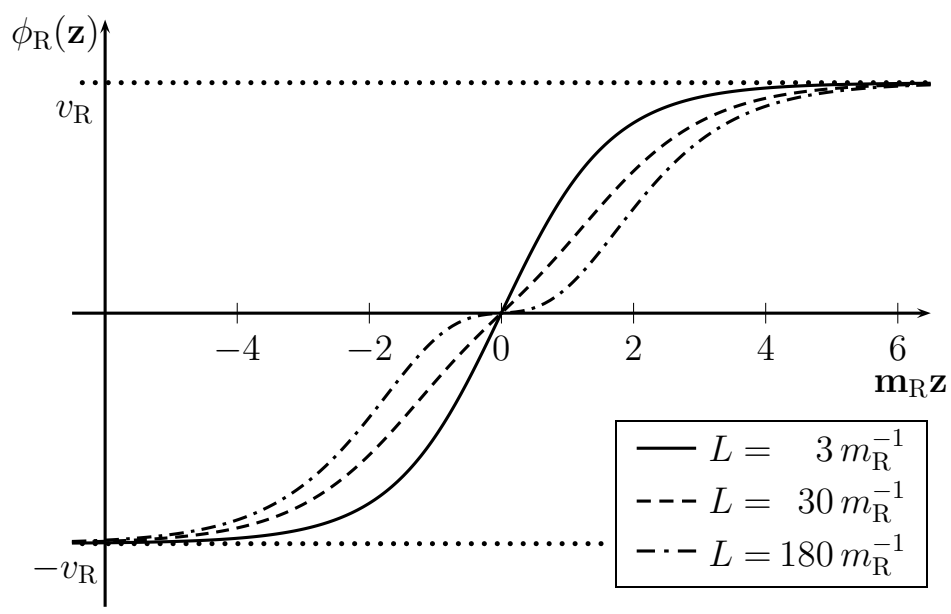

Figure 1: The renormalized interface profile for different system sizes at a coupling of $u_{R}=14.3$. 
For $L$ larger than $200 \xi$ the profile is no longer a monotonic function of $z$. In this region the one-loop contribution approaches values of the size of the leading order term and the one-loop approximation reaches its limit of validity.

A three-dimensional calculation of the interface profile by field theoretical methods has been performed previously by Jasnow and Rudnick 19] in the one-loop approximation. They do not consider systems of a finite extent $L$, but use an external gravitational field in order to control capillary wave fluctuations.

Their result for the interface profile has the functional form of Eq. (52), with coefficients that are given numerically. In place of our term

$$
-\frac{u_{R}}{16 \pi} \ln \left(m_{R} L\right) \tanh \left(\frac{m_{R}}{2} z\right) \operatorname{sech}^{2}\left(\frac{m_{R}}{2} z\right)
$$

they get

$$
c_{3} \ln (h) \tanh \left(\frac{m_{R}}{2} z\right) \operatorname{sech}^{2}\left(\frac{m_{R}}{2} z\right)
$$

where $h$ is the external field, and $c_{3}=0.109975$. As this term originates from long-wavelength fluctuations, its coefficient can be expected to correspond to ours. For a comparison one has to take into account that $h$ corresponds to $L^{-2}$ 1]. Also, they use an estimate of the fixed point value of the coupling which is slightly different from ours. In view of this, the numerical coefficient $c_{3}$ is in rough agreement with our result.

The coefficients of the other terms are different from ours. We do not know, whether there is reason to expect them to be the same.

\section{$5 \quad$ Interface width}

There are various ways to define the width $w$ of an interface, see e.g. refs. [9, [8] and [29. A suitable choice is

$$
w^{2}=\left\langle z^{2}\right\rangle=\int d z z^{2} p(z)
$$

where the weight $p(z)$ is taken to be proportional to the square of the gradient of the profile,

$$
p(z) \propto\left(\partial_{z} \phi_{R}(z)\right)^{2}
$$

and to be normalized:

$$
\int d z p(z)=1
$$

In the evaluation of $w^{2}$ it should be observed that the occurring terms have to be expanded consistently in powers of the coupling. For example, in the 
one-loop approximation, the square of the gradient $\partial_{z} \phi=\partial_{z} \phi_{0}+\partial_{z} \phi_{1}$ is to be taken as

$$
\left(\partial_{z} \phi\right)^{2}=\left(\partial_{z} \phi_{0}\right)^{2}+2 \partial_{z} \phi_{0} \partial_{z} \phi_{1}+\mathcal{O}\left(u_{R}^{2}\right) .
$$

With the interface profile given above, we obtain

$$
w^{2}=\frac{b}{m_{R}^{2}}+\frac{3 u_{R}}{20 \pi m_{R}^{2}} \ln \left(m_{R} L\right)
$$

with

$$
b=\frac{\pi^{2}-6}{3}-\frac{u_{R}}{16 \pi}\left[\frac{12}{5} \alpha-\left(\pi^{2}-6\right)\left(\ln 3-\frac{13}{12}\right)\right] .
$$

The interface width grows logarithmically with the system size. So the field theoretic calculation in the one-loop approximation confirms the prediction of capillary wave theory. Our result, however, does not rely on the capillary wave approximation, but comes from taking into account fluctuations of the density profile on all scales. Moreover, the numerical coefficients are fixed unambiguously and do not depend on ad hoc cut-offs.

For a direct comparison with the convolution approximation another definition of the interface width is more convenient, namely choosing

$$
p(z)=\left(2 v_{R}\right)^{-1} \partial_{z} \phi_{R}(z),
$$

which is meaningful as long as the profile function is monotonic. For this choice, in the convolution approximation the squared width $\tilde{w}^{2}$ of the interface equals the sum of the intrinsic and the capillary wave contributions, see Eq. (4).

With this definition of the interface width, our result reads

$$
\tilde{w}^{2}=\frac{a}{m_{R}^{2}}+\frac{u_{R}}{4 \pi m_{R}^{2}} \ln \left(m_{R} L\right),
$$

with

$$
a=\frac{\pi^{2}}{3}-\frac{u_{R}}{16 \pi}\left\{4 \alpha-\pi^{2}\left(\ln 3-\frac{13}{12}\right)\right\}=1.249 .
$$

By noting that for the interface tension we have [16

$$
\frac{1}{\sigma}=\frac{u_{R}}{2 m_{R}^{2}}+\mathcal{O}\left(u_{R}^{2}\right),
$$

we see that the $L$-dependent term is in agreement with the prediction (3) from the capillary wave model.

The $L$-independent term $a$ contains the classical mean field value

$$
a_{0}=\frac{\pi^{2}}{3}
$$


see [32, plus corrections, which are undetermined in the convolution approximation.

For both choices of the weight function $p(z)$, the one-loop approximation ceases to be valid, if $L$ gets so large that $p(z)$ becomes negative. This happens for $L \approx 200 \xi$, which coincides with the value, where the profile begins to be non-monotonic.

Interfaces have been investigated in the three-dimensional Ising model by means of Monte Carlo calculations in 30, 29, 31, 32. To observe roughening in Monte Carlo is delicate, nevertheless one can obtain estimates for the offset $a$ from their data, which amount to $a=2.68$ [30], $a=0.76$ [29], $a=3.4431$ and $a=0.08$ 32. In view of the spread of these numbers, and in view of the fact that higher-loop contributions will change our estimate, we can only notice that the order of magnitude is compatible with our result.

\section{Conclusion}

Field theory, in the form of the Landau-Ginzburg model, including thermal fluctuations on all length scales, allows to determine the interface profile and interface width for models in the Ising universality class in the critical region. For a system possessing a square interface of size $L \times L$ we derived the conditional equation for the interface profile in the one-loop approximation. We obtained its solution in analytical form. When it is expressed in terms of physical, renormalized parameters, no divergences occur, and there is no need to introduce ad hoc cut-offs as in the capillary wave model.

The solution displays the characteristics of roughening by depending logarithmically on the size $L$. The interface width grows logarithmically with increasing system size. The coefficient of the logarithmic term is in agreement with the universal part of the capillary wave model, and the constant term is consistent with results from Monte Carlo simulations of the Ising model.

\section{References}

[1] J. Rowlinson and B. Widom, Molecular Theory of Capillarity (Clarendon Press, Oxford, 1982).

[2] J. S. Huang and W. W. Webb, Viscous damping of thermal excitations on the interface of critical fluid mixtures, Phys. Rev. Lett. 23: 160-163 (1969).

[3] D. Langevin, ed., Light Scattering by Liquid Surfaces and Complementary Techniques (Dekker, New York, 1992). 
[4] R. K. Thomas, Neutron reflection from liquid interfaces, Annual Review of Physical Chemistry 55: 91-426 (2004).

[5] A. Werner, F. Schmid, M. Müller and K. Binder, Anomalous sizedependence of interfacial profiles between coexisting phases of polymer mixtures in thin film geometry: A Monte-Carlo simulation, J. Chem. Phys. 107: 8175-8188 (1997).

[6] A. Werner, F. Schmid, M. Müller and K. Binder, "Intrinsic" profiles and capillary waves at homopolymer interfaces: A Monte Carlo study, Phys. Rev. E 59: 728-738 (1999).

[7] B. Dünweg, D. P. Landau and A. Milchev, eds., Computer Simulations of Surfaces and Interfaces (Kluwer Acad. Publ., Dordrecht, 2003).

[8] F. Buff, R. Lovett and F. Stillinger, Interfacial density profile for fluids in the critical region, Phys. Rev. Lett. 15: 621-623 (1965).

[9] D. Jasnow, Critical phenomena at interfaces, Rep. Prog. Phys. 47: 1059-1132 (1984).

[10] B. Widom, in Phase Transitions and Critical Phenomena, Vol. 2, C. Domb and M. Green, eds. (Academic Press, New York, 1972).

[11] T. Ohta and K. Kawasaki, Renormalization group approach to the interfacial order parameter profile near the critical point, Prog. Theor. Phys. 58: 467-481 (1977).

[12] J. Rudnick and D. Jasnow, $\epsilon$ expansion for the interfacial profile, Phys. Rev. B 17: 1351-1354 1978.

[13] J. H. Sikkenk and J. M. J. van Leeuwen, $\epsilon$-expansion for the interfacial profile in an external field, Physica A 137: 156-177 (1986).

[14] G. Parisi, Field-theoretic approach to second-order phase transition in two-and three-dimensional systems, J. Stat. Phys. 23: 49 - 82 (1980).

[15] J. C. Le Guillou and J. Zinn-Justin, Critical exponents from field theory, Phys. Rev. B 21: 3976 - 3998 (1980).

[16] G. Münster, Interface tension in three-dimensional systems from field theory, Nucl. Phys. B 340: 559-567 (1990).

[17] G. Münster and J. Heitger, Field-theoretic calculation of the universal amplitude ratio of correlation lengths in $3 D$ Ising systems, Nucl. Phys. $B$ 424: 582-594 (1994). 
[18] C. Gutsfeld, J. Küster and G. Münster, Calculation of universal amplitude ratios in three-loop order, Nucl. Phys. B 479: 654-662 (1996).

[19] D. Jasnow and J. Rudnick, Interfacial profile in three Dimensions, Phys. Rev. Lett. 41: 698-701 (1978).

[20] M. Le Bellac, Quantum and Statistical Field Theory (Clarendon Press, Oxford, 1991).

[21] J. van der Waals, The thermodynamic theory of capillarity under the hypothesis of a continuous variation of density, Verhandel Konink. Akad. Weten. 1 (1893). English translation: J. Rowlinson, J. Stat. Phys. 20: 97-244 (1979).

[22] J. Cahn and J. Hilliard, Free energy of a nonuniform system, J. Chem. Phys. 28: 258-267 (1958).

[23] R. Rajamaran, Non-perturbative semi-classical methods in quantum field theory (a pedagogical review), Phys. Rep. 21 C: 227-313 (1975).

[24] J. L. Gervais and B. Sakita, Extended particles in quantum field theories, Phys. Rev. D 11: 2943-2945 (1975).

[25] J. Küster and G. Münster, The interfacial profile in two-loop order, $J$. Stat. Phys. 129: 441-451 (2007).

[26] M. Lüscher and P. Weisz, Scaling laws and triviality bounds in the lattice $\phi^{4}$-theory; one-component model in the phase with spontaneous broken symmetry, Nucl. Phys. B 295: 65-92 (1987).

[27] S. Fisk and B. Widom, Structure and free energy of the interface between fluid phases in equilibrium near the critical point, J. Chem. Phys. 50: 3219-3227 (1960).

[28] M. Caselle and M. Hasenbusch, Universal amplitude ratios in the 3D Ising model, J. Phys. A 30: 4963-4982 (1997).

[29] K. Mon, D. Landau and D. Stauffer, Interface roughening in the threedimensional Ising model, Phys. Rev. B 42: 545-547 (1990).

[30] E. Bürkner and D. Stauffer, Monte Carlo study of surface roughening in the three-dimensional Ising model, Z. Phys. B 53: 241-243 (1983).

[31] M. Hasenbusch and K. Pinn, Surface tension, surface stiffness, and surface width of the 3 -dimensional Ising model on a cubic lattice, Physica A 192: 342-374 (1992).

[32] M. Müller and G. Münster, Profile and width of rough interfaces, $J$. Stat. Phys. 118: 669-686 (2005). 